\title{
Postoperative discomfort and emergence delirium in children undergoing dental rehabilitation under general anesthesia: comparison of nasal tracheal intubation and laryngeal mask airway
}

This article was published in the following Dove Press journal: Journal of Pain Research

\author{
Sultan Keles' \\ Ozlem Kocaturk ${ }^{2}$ \\ 'Department of Pediatric Dentistry, \\ ${ }^{2}$ Deparment of Oral and Maxillofacial \\ Surgery, Division of Anesthesiology, \\ Faculty of Dentistry, Adnan Menderes \\ University, Aydin, Turkey
}

Background: Several studies have identified side effects of general anesthesia with endotracheal intubation, such as laryngeal pain, dysphonia, and postoperative nausea and vomiting (PONV). The laryngeal mask airway (LMAw) is believed to decrease these side effects. The aim of this trial was to compare postoperative discomfort, emergence delirium, and recovery time of patients who received either an LMAw or nasotracheal intubation (NTI).

Patients and methods: A total of 70 children were randomly assigned to the LMAw group $(n=35)$ or the NTI group ( $n=35)$. Both groups underwent mask induction with $8 \%$ sevoflurane. The NTI group received muscle relaxant, whereas the LMAw group did not. Postoperative laryngeal pain, dental pain, dysphonia, and PONV were assessed immediately and at 1 hour and 6 hours postoperatively. The Wong-Baker Faces Scale was used to evaluate the patients' self-reported pain. In addition, decayed, missing, and filled teeth (dft/DMFT) values, dental procedure type, number of dental procedures, duration of the dental operation, duration of anesthesia, recovery time, emergence delirium, pediatric dentist's access to the mouth, and parents' satisfaction levels were recorded. Data were analyzed using descriptive statistics, chisquare tests, and two-sample $t$-tests.

Results: The incidence of postoperative laryngeal pain was significantly higher in the NTI group immediately $(97.2 \%$ vs. $8.5 \%, P=0.00), 1$ hour $(94.2 \%$ vs. $0 \%, P=0.00)$, and 6 hours postoperatively $(25.7 \%$ vs. $0 \%, P=0.00)$. There were no statistically significant differences between the two groups in dental pain scores, dft/DMFT values, duration of anesthesia, duration of the dental operation, number of dental procedures, the incidence of PONV, or pediatric dentist's access to the mouth $(P>0.05)$. Emergence delirium and recovery time were significantly higher in the NTI group $(P<0.05)$.

Conclusion: The LMAw provided a more comfortable postoperative period than NTI for children who underwent full-mouth dental rehabilitation under general anesthesia.

Keywords: anesthesia, emergence delirium, pain, intubation, child, dysphonia

\section{Introduction}

General anesthesia is frequently used for dental procedures when young children are unable to cooperate or when a surgical procedure is required. General anesthesia may also be used for dental procedures if the patient has a strong gag reflex, a mental disability, or a significant amount of fear and anxiety. ${ }^{1,2}$ General anesthesia provides efficient and convenient treatment and exerts less physical and mental stress on both the patients and parents. There are some disadvantages of general anesthesia, however,
Department of Pediatric Dentistry, Faculty of Dentistry, Adnan

Menderes University Hasan Efendi St, Aydin 09100, Turkey

Tel +902562133939

Fax +90256 215 1918

Email dtsultank@gmail.com 
including postoperative nausea and vomiting (PONV), headache, emergence agitation, vertigo, dizziness, and diplopia; more severe complications include postoperative pain, cardiac events, anaphylaxis, laryngospasm, respiratory depression, and respiratory arrest. ${ }^{1}$

Various techniques are used for airway management with general anesthesia for pediatric dental procedures. Nasotracheal intubation (NTI) is commonly used for head and neck surgeries, particularly intraoral procedures. NTI provides unrestricted access to the mouth, enlarges the surgical field, and facilitates the insertion of instruments. ${ }^{3,4}$ However, NTI has some disadvantages, including a slower recovery period and adverse postoperative events such as coughing, laryngeal pain, and dysphonia.,

Due to its ease of insertion and removal, the laryngeal mask airway (LMAw) is a useful alternative to NTI in children. Moreover, the LMAw has a lower risk of upper respiratory tract injury and better patient tolerance. ${ }^{6}$ When airway reflexes are suppressed by an adequate depth of anesthesia, the LMAw can easily be inserted without using a muscle relaxant. Postoperative morbidities such as sore throat and nausea and vomiting are less frequent with the LMAw than with NTI. ${ }^{6}$ Zhao et $\mathrm{al}^{6}$ found that the classic LMAw can provide good surgical conditions, adequate control of the airway, and fewer postoperative complications compared with NTI in children who underwent same-day dental surgery. Radu et $\mathrm{al}^{5}$ also found that use of an endotracheal tube was associated with a higher incidence of laryngeal pain and hoarseness during the first postoperative hours in adults.

Emergence delirium (ED) in children is still considered as a mysterious complication which increases the risk of patient self-harm, places a burden on nursing staff, and reduces parent satisfaction with the treatment during recovery from general anesthesia. Although the exact cause of ED in children is not yet determined, age, preoperative anxiety, patient personality, pain, anesthesia method, and surgical procedures are risk factors for ED. Tian et $\mathrm{al}^{7}$ reported that the incidence of ED in children who had endotracheal intubation (20\%) was higher than the children who had been inserted LMAw (8\%) for various surgical procedures.

The primary aim of this study was to compare the LMAw and NTI in terms of postoperative discomfort and ED in children who underwent full-mouth dental rehabilitation under general anesthesia. Specifically, we investigated the differences between the two methods with respect to postoperative recovery time, ED, and the satisfaction levels of the patients' parents; we also compared the pediatric dentist's access to the patient's mouth with an LMAw vs. NTI.

\section{Patients and methods}

This study was a prospective, randomized controlled trial (RCT) approved by the Dentistry Ethics Committee of Adnan Menderes University (reference no. 2017/011). The trial was also registered (Protocol Registration Receipt NCT03197753) at www.ClinicalTrials.gov. Written informed consent was obtained from the parents of all study participants.

A total of 70 patients were recruited (aged 3-7 years, American Society of Anesthesiologist [ASA] status I and II) who were referred to the Pediatric Dentistry Department of Adnan Menderes University between June 2017 and August 2017 for the treatment of dental decay under general anesthesia. Exclusion criteria included anticipated difficult intubation, pharyngeal pathology, known pulmonary or cardiovascular disease, mental retardation, if the dental procedure was expected to last longer than 2 hours, and if the parents did not consent to the child's participation in the study. Parents were allowed to be present during the induction of anesthesia.

All patients were randomly assigned to one of the two groups, such that each group contained 35 patients. Patients were assigned to a group based on a closed, coded, opaque envelope chosen by a ward nurse on the day of the operation, and the randomization process was supervised by a single author.

\section{Anesthesia protocol and groups NTI group}

All patients in the NTI group were intubated by the same (OK) experienced anesthesiologist (nearly 15 years of experience). Mask induction was carried out with $8 \%$ sevoflurane and $100 \%$ oxygen. Following the loss of consciousness, an intravenous (i.v.) line was established through which $2 \mathrm{mg} / \mathrm{kg}$ of $1 \%$ propofol (Propofol-Lipuro ${ }^{\circledR}$; B. Braun Melsungen AG, Germany), $1 \mu \mathrm{g} / \mathrm{kg}$ of fentanyl (Talinat ${ }^{\circledR}$; Vem, Istanbul, Turkey), and $0.5 \mathrm{mg} / \mathrm{kg}$ of rocuronium (Myocron ${ }^{\circledR}$; Vem) were administered, followed by NTI with a cuffed tube. The nasotracheal tube was selected using the following formula: tube size $=([$ age $/ 4]+3.5)$. The surgeon placed the tongue blade when sufficient anesthetic depth had been achieved.

\section{LMAw group}

After performing mask induction with $8 \%$ sevoflurane and $100 \%$ oxygen, a laryngeal mask was lubricated with water- 
soluble jelly and inserted into the airway by the same anesthesiologist (OK). The size of the LMAw was chosen based on the child's weight (LMAw sizes 1.5, 2, and 2.5 were used for children weighing 5-10, 10-20, and 20-30 kg, respectively). After insertion, the LMAw was secured and inflated according to the manufacturer's instructions. No muscle relaxant was used in the LMAw group. Anesthesia was maintained with $2 \%$ sevoflurane in a 50:50 mixture of oxygen and nitrous oxide. All children received $0.4 \mathrm{mg} / \mathrm{kg}$ of i.v. tenoxicam (Tilcotil ${ }^{\circledR}$; Deva, Istanbul, Turkey) for analgesia and $0.4 \mathrm{mg} /$ $\mathrm{kg}$ of i.v. metoclopramide for PONV prophylaxis 15 minutes prior to the end of surgery. ${ }^{8}$ Standard monitoring including noninvasive arterial blood pressure, electrocardiography, respiratuar rate, end-tidal $\mathrm{CO}_{2}$, and $\mathrm{SpO}_{2}$ was placed at the operating room for both of the groups.

\section{Dental treatment procedures}

All the procedures were performed by the same pediatric dentist (SK). Following intubation, the nasotracheal tube was secured with medical tape, and a mouth probe and a pharyngeal pack were inserted. In the LMAw group, the LMAw was secured to the corner of the mouth after the mouth probe was inserted opposite to the planned operative site. After completion of the procedures on one side of the mouth, the LMAw was secured to the other side of the mouth. Decayed teeth underwent either restorative (compomer resin, composite) or endodontic (pulpotomy, pulpectomy) procedures. Teeth that could not be restored were extracted.

The number of dental procedures, duration of the dental operation, and duration of anesthesia were noted. Once all procedures were completed, LMAw or endotracheal tube was removed after regaining protective airway reflexes. Patients were then transferred to the post-anesthesia care unit (PACU).

In the PACU, patients were evaluated at 5-minute intervals with the Aldrete scale. The Aldrete scoring system is used to clinically evaluate the physical status of patients recovering from general anesthesia. A score of 0-2 is given for each of five categories (activity, circulation, consciousness, $\mathrm{O}_{2}$ saturation, and respiration) for a maximum score of $10 .{ }^{9}$ Recovery time was calculated from the patient's arrival to the PACU until the Aldrete score was $\geq 9$ and the child could answer the nurse.

\section{Outcome measures}

A research nurse, who was blinded to the airway management method, recorded the data regarding postoperative discomfort. The following outcome parameters were recorded immediately (at the end of the recovery time in PACU), and at 1 hour and 6 hours postoperatively: laryngeal pain, dental pain, dysphonia, PONV, recovery time, and the Pediatric Anesthesia Emergence Delirium (PAED) scale. In addition, the pediatric dentist's access to the mouth and the parents' or guardians' satisfaction levels were recorded.

Patient pain (laryngeal and dental) was self-assessed using the Wong-Baker Faces Scale immediately and at 1 hour and 6 hours postoperatively. Dysphonia was evaluated via the dysphonia scale: 0 , no dysphonia; 1 , mild dysphonia; 2, moderate dysphonia; 3, severe dysphonia; and 4, aphonia; patients were then dichotomized with either the presence of dysphonia (a score of 1, 2, 3, or 4) or the absence of dysphonia (score of 0 ).

The PAED scale that was a reliable tool to measure ED was used in this study. The PAEDS involves five items: eye contact, purposeful actions, awareness of the surroundings, restlessness, and inconsolability was also used after discharge from the PACU. A PAED scale score of $\geq 10$ signified the presence of ED (Box 1). ${ }^{10}$ Patients were aditted to the pediatric dental service after being discharged from the PACU. The parents or guardians were asked to grade their satisfaction levels from 0 to 10 ( 0 , very dissatisfied; 10 , very satisfied). The pediatric dentist also graded the surgical access to the mouth as excellent, obstructed view but able to operate, or poor.

\section{Statistical analysis}

The sample size ( 35 patients per group) was determined by the Gpower software based on a study by Zhao et $\mathrm{al}^{6}$ to detect large effects $(\mathrm{d}=0.08)$ with a power of $95 \%$ using a Student's $t$-test to compare mean with an alpha value of 0.05 . All data were saved in a Microsoft Excel database and exported to the Statistical Package for the Social Sciences program for Windows (SPSS 20.0; IBM Corporation, Armonk, NY, USA) for statistical analysis. All data were subjected to the Kolmogorov-Smirnov test for normality. Two-sample $t$-tests were used to compare age, weight, duration of the operation, duration of anesthesia, number of dental procedures, recovery time, and decayed,

\section{Box I The PAEDS devised by Sikich and Lerman ${ }^{10}$}

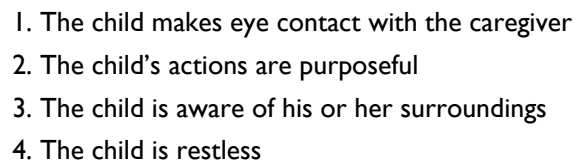

Notes: Items I, 2, 3 are reversed scored as follows: $4=$ not at all, $3=$ =just a little, 2= quite a bit, $\mathrm{I}=$ very much, $0=$ extremely. Items 4 and 5 are scored as follows $0=$ not at all, I= just a little, $2=$ quite a bit, $3=$ very much, $4=$ extremely. The scores of each item are summed to obtain a total PAEDS score. ED increases directly with the total score. A score of $\geq 10$ was considered as the presence of ED. Adapted from Sikich $\mathrm{N}$, Lerman J. Development and psychometric evaluation of the pediatric anaesthesia emergence delirium scale. Anesthesiology. 2004; I00(5): I I 38-1 I 45. ${ }^{10}$

Abbreviations: ED, emergence delirium; PAEDS, Pediatric Anesthesia Emergence Delirium Scale. 
missed, and filled teeth (dft/DMFT) values between the two groups. Chi-square tests were used to compare categorical variables between the two groups. A 5\% type 1 error level was used to determine statistical significance.

\section{Results}

The study sample consisted of 35 boys and 35 girls with a mean age of $4.6 \pm 1.1$ years (Table 1). Figure 1 represents recruitment of the patients and follow-up. There were no significant differences in age, gender, weight, dft/DMFT values, duration of operation, duration of anesthesia, or number of dental procedures between the two groups $(P>0.05$; Table 1$)$.

There was a statistically significant difference between the groups in terms of laryngeal pain, with a higher incidence in the NTI group immediately ( $97.2 \%$ vs. $8.5 \%, P=0.00)$, 1 hour $(94.2 \%$ vs. $0 \%, P=0.00)$, and 6 hours postoperatively $(74.2 \%$ vs. $0 \%, P=0.00$; Figure 2 ). There was no statistically significant difference between the groups in dental pain scores $(P>0.05)$. Figure 2 shows the comparison of the incidence of laryngeal and dental pain immediately and at 1 hour and 6 hours postoperatively.

The presence of dysphonia was significantly higher in the NTI group than in the LMAw group immediately $(91.4 \%$ vs. $\left.14.2 \%, x^{2}=41.79, P=0.00\right), 1$ hour $(45.7 \%$ vs. $5.7 \%$, $\left.x^{2}=14.65, P=0.00\right)$, and 6 hours postoperatively $(34.2 \%$ vs. $2.8 \%, x^{2}=11.4, P=0.00$; Table 2).

There was no significant difference between the groups in the incidence of PONV $(P>0.05)$.

Table I Demographic profile, operation times, and dft/DMFT values of the patients

\begin{tabular}{llll}
\hline $\begin{array}{l}\text { Demographic and clinical } \\
\text { characteristics }\end{array}$ & Group NTI $(\mathbf{n = 3 5 )}$ & Group LMAw $(\mathbf{n}=\mathbf{3 5})$ & $\mathbf{x}^{2} / \mathbf{t} / \mathbf{P}$-value \\
\hline Age (years) & $4.4 \pm 1.1$ & $4.8 \pm 1.2$ & $-/-1.62 / 0.11$ \\
Gender: male/female ${ }^{\mathrm{a}}$ & $17 / 18$ & $18 / 17$ & $0.05 / 0.81$ \\
Weight $(\mathrm{kg})$ & $17.7 \pm 3.5$ & $19.0 \pm 4.6$ & $-/-1.235 / 0.22$ \\
Duration of operation $(\mathrm{min})$ & $68.2 \pm 24.0$ & $63.3 \pm 20.9$ & $-/ 0.90 / 0.36$ \\
Duration of anesthesia $(\mathrm{min})$ & $87.4 \pm 24.3$ & $63.3 \pm 20.9$ & $-/ 0.88 / 0.38$ \\
dft/DMFT & $11.2 \pm 3.7$ & $9.8 \pm 3.4$ & $-/ 1.50 / 0.13$ \\
\hline
\end{tabular}

Notes: ${ }^{a}$ Data are presented as the number of patients. Values are expressed as mean \pm SD.

Abbreviations: $\mathrm{dft} / \mathrm{DMFT}$, decayed, missing, and filled teeth; LMAw, laryngeal mask airway; NTI, nasotracheal intubation.

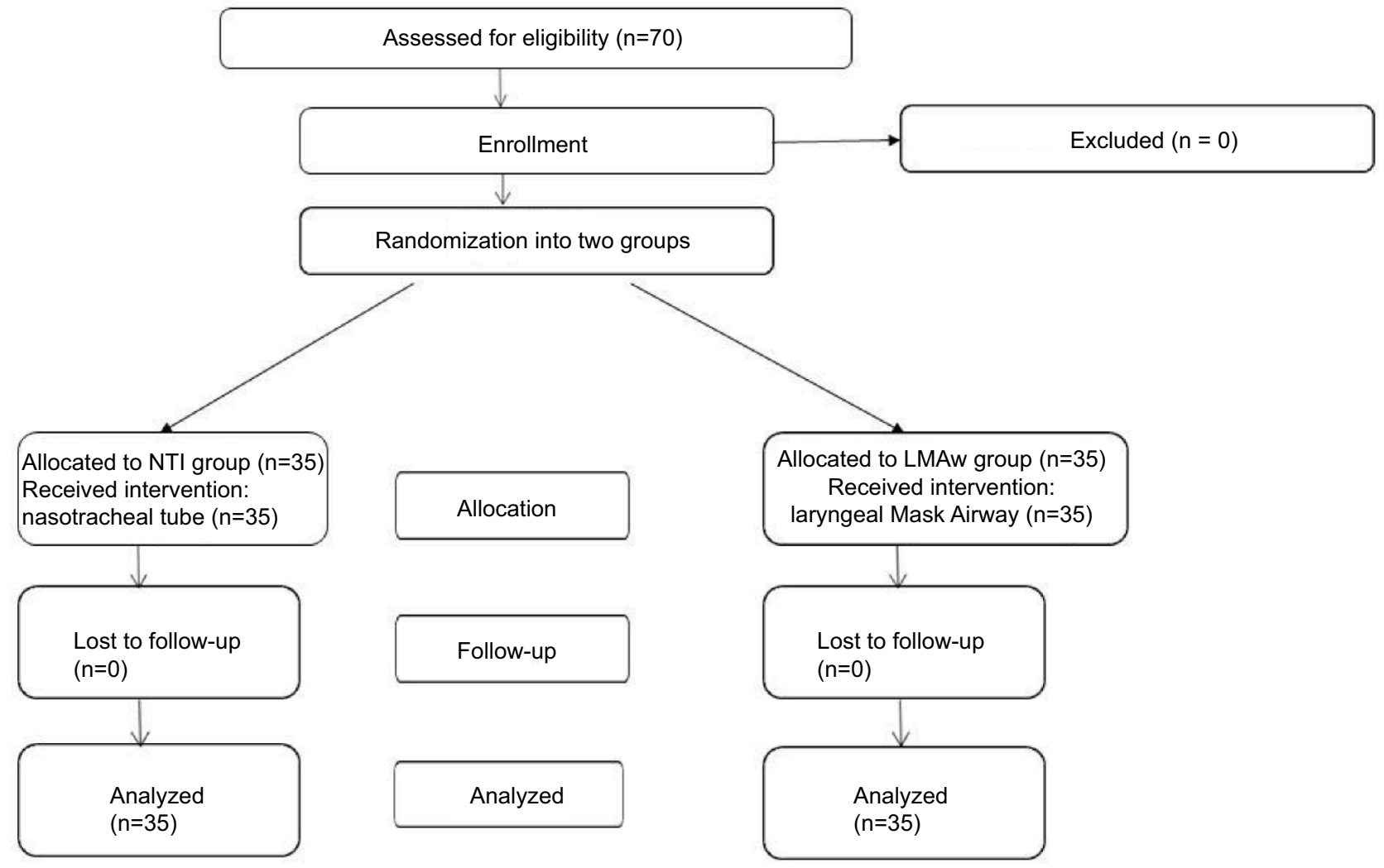

Figure I Flowchart of the study.

Abbreviations: LMAw, laryngeal mask airway; NTI, nasotracheal intubation. 


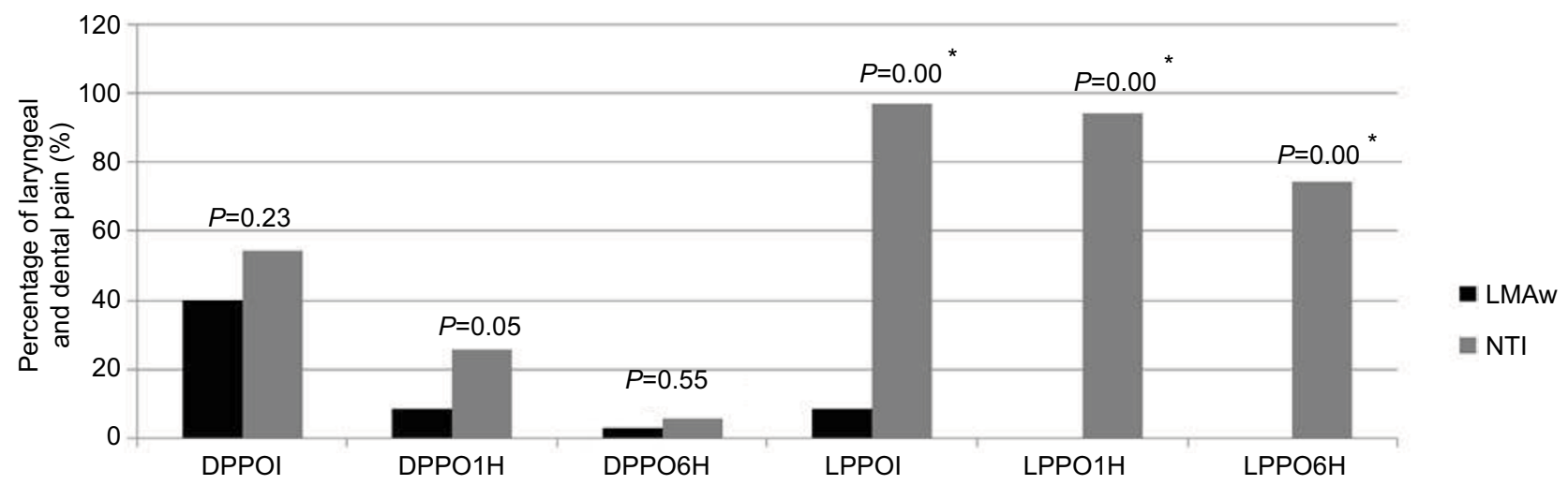

Figure 2 Comparison of the dental and laryngeal pain levels of the groups immediately and at I hour and 6 hours postoperatively. Note: *Statistically significant difference at 0.05 level.

Abbreviations: DPPOIH, dental pain postoperatively I hour; DPPO6H, dental pain postoperatively 6 hours; DPPOI, dental pain postoperatively immediately; LMAw, laryngeal mask airway; LPPOIH, laryngeal pain postoperatively I hour; LPPO6H, laryngeal pain postoperatively 6 hours; LPPOI, laryngeal pain postoperatively immediately; $\mathrm{NTI}$, nasotracheal intubation.

Table 2 Comparison of postoperative discomforts, dentist's access to the oral cavity, dental procedure, parental satisfaction and recovery time of the groups

\begin{tabular}{|c|c|c|c|}
\hline & Group NTI, n (\%) & Group LMAw, n (\%) & $x^{2} / t / P$-value \\
\hline \multicolumn{4}{|l|}{ ED } \\
\hline Present & $35(100)$ & $2(5.7)$ & \\
\hline Absent & $0(0)$ & $33(94.3)$ & $62.432 /-10.00 *$ \\
\hline \multicolumn{4}{|l|}{ Dysphonia } \\
\hline $\mathrm{PI}$ & $32(91.4)$ & $5(\mid 4.2)$ & $41.79 /-10.00 *$ \\
\hline $\mathrm{PIH}$ & $16(45.7)$ & $2(5.7)$ & $\mid 4.65 /-10.00 *$ \\
\hline $\mathrm{P} 6 \mathrm{H}$ & $12(34.2)$ & $\mathrm{I}(2.8)$ & $11.4 /-/ 0.00^{*}$ \\
\hline \multicolumn{4}{|l|}{ PDA } \\
\hline Excellent & $26(74.3)$ & $22(62.8)$ & I.06/-/0.44 \\
\hline Obstructing view but able to work & $9(25.7)$ & $13(37.2)$ & \\
\hline Poor & $0(0)$ & $0(0)$ & \\
\hline \multicolumn{4}{|l|}{ NDP } \\
\hline Tooth filling & $8.0 \pm 3.4$ & $6.6 \pm 2.8$ & $-/ 1.81 / 0.36$ \\
\hline Pulpotomy & $1.3 \pm 1.1$ & $0.7 \pm 0.9$ & $-/ 2.46 / 0.14$ \\
\hline Pulpectomy & $1.0 \pm 1.5$ & $1.8 \pm 1.6$ & $-/-2.11 / 0.34$ \\
\hline Extraction & $3.1 \pm 2.5$ & $3.2 \pm 2.7$ & $-I-0.90 / 0.40$ \\
\hline $\mathrm{RT}(\mathrm{min})$ & $12.9 \pm 2.8$ & $10.0 \pm 3.6$ & $-/ 3.77 / 0.00^{*}$ \\
\hline PSL $(0-10)$ & $6.2 \pm 1.4$ & $8.2 \pm 1.4$ & $-1-5.80 / 0.00 *$ \\
\hline
\end{tabular}

Note: *Statistically significant difference at $P<0.05$ level.

Abbreviations: ED, emergence delirium; LMAw, laryngeal mask airway; NDP, number of dental procedures; NTI, nasotracheal intubation; PDA, pediatric dentist's access; $\mathrm{PIH}$, postoperative I hour; P6H, postoperative 6 hours; PI, postoperative immediately; PSL, parental satisfaction level; RT, recovery time.

The pediatric dentist's access to the mouth was not significantly different between two groups $\left(x^{2}=1.06, P=0.44\right)$. Recovery time was significantly shorter in the LMAw group compared with the NTI group ( $t=3.77, P=0.00$; Table 2).

In the PACU, children in the LMAw group showed significantly lower ED scores compared with the NTI group $\left(x^{2}=62.43, P=0.00\right)$. Parents' global satisfaction scores were also significantly higher in the LMAw group than the NTI group $(P<0.05$; Table 2$)$.

\section{Discussion}

This prospective RCT showed that there was less postoperative discomfort with an LMAw compared with NTI in children who underwent full-mouth dental rehabilitation under general anesthesia. Furthermore, the LMAw did not significantly interfere with the treatment more than NTI. In addition, recovery time was lower and parents' satisfaction levels were higher in the LMAw group compared with the NTI group. 
General anesthesia is a technique that allows pediatric dentists to treat patients who have significant fear and anxiety as well as younger uncooperative patients. By reducing the patient's anxiety and movement with general anesthesia, pediatric dentists can provide significantly better dental care. ${ }^{11}$ General anesthesia via endotracheal intubation for dental procedures may be performed using either an oral or nasal approach. Nasal intubation has many advantages over oral intubation such as improved working space in the oral cavity and the ability to evaluate jaw relationships. ${ }^{12}$ Compared with endotracheal intubation, the LMAw may reduce the incidence of postoperative side effects such as pain, nausea and vomiting, coughing, and dysphonia. ${ }^{5}$ The LMAw is an alternative to NTI that can control the upper airway with less risk of trauma to the larynx. Jamil et al ${ }^{13}$ concluded that the incidence of postoperative soft tissue trauma due to intubation in children between the age range of 2 and 10 years was higher in endotracheal intubation (10\%) than LMAw (2\%). However, the placement of an LMAw in the mouth can obstruct the surgical field similar to oral endotracheal intubation. Although the pediatric dentist's access to the oral cavity was scored as 'excellent' more often in the NTI group than in the LMAw group, this difference was not statistically significant. In the current study, no significant difference was observed between the number of restorative treatments applied or the duration of the operation between the two groups, indicating that the LMAw did not make it more difficult to work within the mouth.

Zhao et $a l^{6}$ determined that the dentists' access to the mouth and the duration of the operation were not significantly different between patients who received an LMAw vs. endotracheal intubation. However, they did not record the types or numbers of dental procedures in their study.

In this study, we focused on postoperative discomfort, ED, and recovery time after using two different airway management techniques. Laryngeal pain was significantly higher in the NTI group than in the LMAw group; however, there was no significant difference in postoperative dental pain scores between the two groups.

Previous studies have shown that the type of dental treatment administered under general anesthesia significantly impacts postoperative dental pain. ${ }^{14}$ In our study, no significant difference was found between the postoperative dental pain scores in the LMAw and NTI groups, most likely because there was no significant difference between the types and number of dental treatment procedures performed between the groups. Needleman et $\mathrm{al}^{15}$ found that NTI was more often associated with sore throat than orotracheal intubation following general anesthesia for dental surgery; children whose tracheal intubations were traumatic were also more likely to report postoperative sore throat. In our study, the incidence of postoperative laryngeal pain was $74.2 \%$ for the NTI group and $0 \%$ for the LMAw group 6 hours postoperatively. Similar to the results of our study, many studies have shown that laryngeal pain is more frequent in patients who receive NTI compared with an LMAw. Radu et $\mathrm{al}^{5}$ observed a significant difference between orotracheal intubation and LMAw groups in terms of pharyngolaryngeal pain with a higher incidence in the orotracheal intubation group. In their study, the incidence of pharyngolaryngeal pain with orotracheal intubation 6 and 24 hours postoperatively was $74 \%$ and $27 \%$, respectively; the incidence of pharyngolaryngeal pain with an LMAw 6 and 24 hours postoperatively was $27 \%$ and $0 \%$, respectively. Seung and Beirne ${ }^{16}$ compiled the results of 29 RCTs comparing endotracheal intubation and LMAw in general anesthesia and found that the incidence of postoperative sore throat was $21.5 \%$ for the LMAw and $34.3 \%$ for the endotracheal intubation. Because the LMAw did not obstruct the surgical field in the mouth or interfere with treatment in the current study, we believe it is a good choice for pediatric dental procedures.

Endotracheal intubation has been associated with dysphonia following general anesthesia, although the reported incidence varies widely. ${ }^{17,18}$ The LMAw, however, has been shown to have a lower incidence of postoperative voice problems. Abdi et al ${ }^{19}$ compared the incidence of postoperative dysphonia in patients who underwent laparoscopic gynecological surgery with either endotracheal intubation or an LMAw and found a higher incidence of dysphonia in the intubated group. Lalwani et al ${ }^{18}$ found that there was no dysphonia after removal of an LMAw or endotracheal tube in patients who underwent elective ophthalmological or lower abdominal surgery. In our study, there was a higher incidence of dysphonia in the NTI group compared with the LMAw group, which was similar to previous studies.

Risk factors for PONV include preschool age (3-6 years), duration of anesthesia, type of surgery (tympanoplasty, tonsillectomy, strabismus surgery, and orchiopexy are considered high risk), predisposition to PONV, multiple doses of opioid, and volatile anesthetic agents..$^{20,21}$ Whether the airway management technique affects PONV is still being debated. Zhao et $\mathrm{al}^{6}$ and Hung et $\mathrm{al}^{22}$ concluded that there was a higher incidence of PONV with endotracheal intubation compared with an LMAw in patients who underwent dental procedures. Radu et $\mathrm{al}^{5}$ found that there was no significant difference between endotracheal intubation and an LMAw in 
terms of nausea and vomiting 6 and 24 hours postoperatively. In our study, we did not find a significant difference between the NTI and LMAw groups in terms of PONV. This may be because there were no significant differences in age, gender, ASA status, type and number of dental procedures, or duration of anesthesia between the two groups.

Muscle relaxant during the induction of anesthesia has been shown to facilitate tracheal intubation, decrease complications associated with endotracheal tube placement, and provide suitable conditions for the operation. ${ }^{23}$ At the same time, the use of muscle relaxation for minor surgery may affect the patient's recovery and prolong the patient's respiratory recovery time. ${ }^{24}$ Laryngoscopy and muscle relaxation are not necessary for the insertion of an LMAw. ${ }^{25}$ The LMAw can even be inserted in awake patients with or without muscle relaxant. In the current study, recovery time in the LMAw group was significantly shorter than the NTI group, which was similar to the results of Zhao et al. ${ }^{6}$ These difference may be due to muscle relaxant not being used in the LMAw group.

ED includes inconsolable crying, thrashing, kicking, disorientation, hallucinations, and cognitive and memory impairment during the recovery period following general anesthesia. The intrinsic characteristics of anesthetic agents, rapid emergence from anesthesia, postoperative pain, preschool age, preoperative anxiety, and child temperament are known risk factors for ED. Preventing ED is important for providing patients with a comfortable postoperative period. In the current study, the incidence of ED was significantly lower in the LMAw group compared with the NTI group. Since there was no significant difference in postoperative dental pain scores between the groups, we believe that this difference in ED may be due to higher laryngeal pain scores observed in the NTI group. Similar to the results of our study, Lee et $\mathrm{al}^{26}$ found that using an LMAw during pediatric surgery reduced the incidence of postoperative emergence agitation compared with tracheal intubation after sevoflurane anesthesia for sub-umbilical surgery. In the current study, parental satisfaction scores were also higher in the LMAw group than in the NTI group. This can be attributed to the lower incidence of postoperative discomfort and ED in the LMAw group.

The main limitation of the current study is the wide age range of the patients, ranging from 3 to 7 years, which could explain the large difference in self-reported pain scores. The second limitation is that the follow-up period was relatively short, as we only evaluated the patients' discomfort during the hospital stay.

\section{Conclusion}

Use of an LMAw resulted in less postoperative discomfort and ED, a shorter recovery time, and higher parental satisfaction levels than NTI. In addition, the LMAw did not obstruct the view during treatment procedures in patients who underwent full-mouth dental rehabilitation.

\section{Disclosure}

The authors report no conflicts of interest in this work.

\section{References}

1. Knapp R, Marshman Z, Rodd H. Treatment of dental caries under general anaesthetic in children. BDJ Team. 2017;4(7):116.

2. Dziedzic A. The role of general anaesthesia in special care \& paediatric dentistry; inclusion criteria and clinical indications. SAAD Dig. 2017;33:47-53.

3. Tsukamoto M, Hitosugi T, Yokoyama T. Flexible laryngeal mask airway management for dental treatment cases associated with difficult intubation. J Dent Anesth Pain Med. 2017;17(1):61-64.

4. Prasanna D, Bhat S. Nasotracheal intubation: an overview. J Oral Maxillofac Surg. 2014;13(4):366-372.

5. Radu AD, Miled F, Marret E, Vigneau A, Bonnet F. Pharyngo-laryngeal discomfort after breast surgery: comparison between orotracheal intubation and laryngeal mask. Breast. 2008;17(4):407-411.

6. Zhao N, Deng F, Yu C. Anaesthesia for pediatric day-case dental surgery: a study comparing the classic laryngeal mask airway with nasal trachea intubation. J Craniofac Surg. 2014;25(3):245-248.

7. Tian Y, Wu XY, Li L, Ma L, Li YF. A clinical trial evaluating the laryngeal mask airway-Supreme in obese children during general anaesthesia. Arch Med Sci. 2017;13(1):183.

8. Tjia I, Dalton M, Watcha MF. Management of postoperative nausea and vomiting in pediatric patients. Postoperative Nausea and Vomiting: a practical guide. Cambridge University Press; 2016:119-130.

9. Aldrete JA, Kroulik D. A postanesthetic recovery score. Anesth Analg. 1970;49(6):924-934.

10. Sikich N, Lerman J. Development and psychometric evaluation of the pediatric anaesthesia emergence delirium scale. Anesthesiology. 2004;100(5):1138-1145.

11. Chen YP, Hsieh CY, Hsu WT, Wu FY, Shih WY. A 10-year trend of dental treatments under general anaesthesia of children in Taipei Veterans General Hospital. J Chin Med Assoc. 2017;80(4):262-268.

12. Wong A, Subar P, Witherell H, Ovodov KJ. Reducing nasopharyngeal trauma: the urethral catheter-assisted nasotracheal intubation technique. Anesth Prog. 2011;58(1):26-30.

13. Jamil SN, Alam M, Usmani H, Khan MM. A study of the use of laryngeal mask airway (LMAW) in children and its comparison with endotracheal intubation. Indian J Anaesth. 2009;53(2):174-178.

14. Ashkenazi M, Blumer S, Eli I. Post-operative pain and use of analgesic agents in children following intrasulcular anaesthesia and various operative procedures. Br Dent J. 2007;202(5):13.

15. Needleman HL, Harpavat S, Wu S, Allred EN, Berde C. Postoperative pain and other sequelae of dental rehabilitations performed on children under general anaesthesia. Pediatr Dent. 2008;30(2):111-121.

16. Seung HY, Beirne OR. Laryngeal mask airways have a lower risk of airway complications compared with endotracheal intubation: a systematic review. J Oral Maxillofac Surg. 2010;68(10):2359-2376.

17. Venugopal A, Jacob RM, Koshy RC. A randomized control study comparing the pharyngolaryngeal morbidity of laryngeal mask airway versus endotracheal tube. Anesth Essays Res. 2016;10(2):189-194.

18. Lalwani J, Dubey KP, Sahu BS, Shah PJ. ProSeal laryngeal mask airway: an alternative to endotracheal intubation in paediatric patients for short duration surgical procedures. Indian J Anaesth. 2010;54(6):541-545. 
19. Abdi W, Amathieu R, Adhoum A, et al. Sparing the larynx during gynecological laparoscopy: a randomized trial comparing the LMAW Supreme ${ }^{\mathrm{TM}}$ and the ETT. Acta Anaesthesiol Scand. 2010;54(2):141-146.

20. Bourdaud N, Devys JM, Bientz J, et al. Development and validation of a risk score to predict the probability of postoperative vomiting in pediatric patients: the VPOP score. Paediatr Anaesth. 2014; 25(3):330.

21. Eberhart LH, Geldner G, Kranke P, et al. The development and validation of a risk score to predict the probability of postoperative vomiting in pediatric patients. Anesth Analg. 2004;99(6):1630-1637.

22. Hung WT, Chen CC, Chau MY, Tsai WY. Effect of reinforced laryngeal mask airway or endotracheal intubation anaesthesia on adverse events: quality of life issues for dental patients requiring general sedation. Spec Care Dentist. 2005;25(4):188-192.
23. Julien-Marsollier F, Michelet D, Bellon M, Horlin A-L, Devys J-M, Dahmani S. Muscle relaxation for tracheal intubation during paediatric anaesthesia: a meta-analysis and trial sequential analysis. Eur J Anaesthesiol. 2017;34(8):550-561.

24. Ledowski T. Muscle relaxation in laparoscopic surgery: what is the evidence for improved operating conditions and patient outcome? A brief review of the literature. Surg Laparosc Endosc Percutan Tech. 2015;25(4): 281-285.

25. Patel B, Patel H, Kantharia B. A comparative study of sevoflurane and propofol for laryngeal mask airway insertion. Int J Med Sci Public Health. 2017;6:295.

26. Lee YC, Kim JM, Ko HB, Lee SR. Use of laryngeal mask airway and its removal in a deeply anaesthetized state reduces emergence agitation after sevoflurane anaesthesia in children. J Int Med Res. 2011;39(6):2385-2392.
Journal of Pain Research

\section{Publish your work in this journal}

The Journal of Pain Research is an international, peer reviewed, open access, online journal that welcomes laboratory and clinical findings in the fields of pain research and the prevention and management of pain. Original research, reviews, symposium reports, hypothesis formation and commentaries are all considered for publication.
Dovepress

The manuscript management system is completely online and includes a very quick and fair peer-review system, which is all easy to use. Visit http://www.dovepress.com/testimonials.php to read real quotes from published authors. 